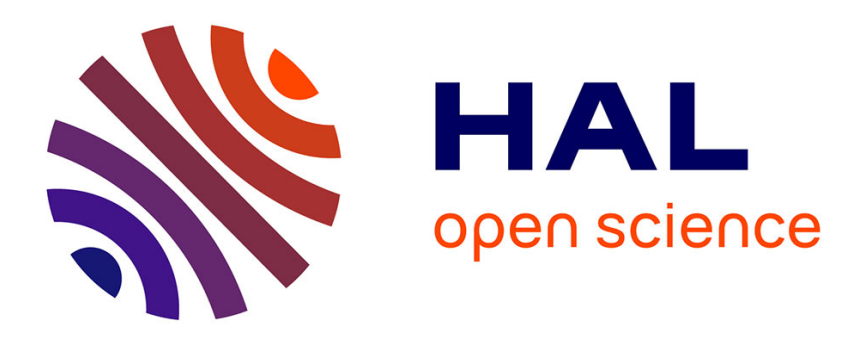

\title{
Nouvelles migrations et districts productifs en Italie
}

Camille Schmoll

\section{To cite this version:}

Camille Schmoll. Nouvelles migrations et districts productifs en Italie. Méditerranée: revue géographique des pays méditerranéens, 2006, 106 (1-2), pp.73-78. halshs-00239269

\section{HAL Id: halshs-00239269 \\ https://shs.hal.science/halshs-00239269}

Submitted on 5 Feb 2008

HAL is a multi-disciplinary open access archive for the deposit and dissemination of scientific research documents, whether they are published or not. The documents may come from teaching and research institutions in France or abroad, or from public or private research centers.
L'archive ouverte pluridisciplinaire HAL, est destinée au dépôt et à la diffusion de documents scientifiques de niveau recherche, publiés ou non, émanant des établissements d'enseignement et de recherche français ou étrangers, des laboratoires publics ou privés. 


\section{Camille Schmoll ${ }^{1}$}

\section{Nouvelles migrations et districts productifs en Italie}

Lôbjectif de ce texte est dôntroduire la question des migrations internationales et la figure de lấtranger au cî ur de la réflexion sur le développement local en Italie, en amont - dans les processus de production - et en aval - en tant que clientèle. Les travaux portant sur ce thème, en effet, nốvoquent que rarement la question des migrations internationales, bien que lâtalie soit devenue un espace dôtccueil depuis plus de deux décennies.

\section{La question du territoire dans les travaux sur le développement local}

Dans ce premier paragraphe, quelques éléments généraux de la littérature sur le développement local en Italie seront présentés, afin dôessayer de comprendre pour quelles raisons les migrants étrangers, devenus aujourdôui des acteurs cruciaux du tissu économique italien, trouvent difficilement leur place dans ces travaux. Il sera vu que lâbsence des migrants est étroitement liée à lâicception de la notion de territoire sur laquelle se fondent ces travaux.

Les analyses sur le développement local en Italie sôppuient sur un constat : il existe une Troisième Italie, localisée principalement en Emilie-Romagne, en Toscane, dans les Marches et en Vénétie. Cette Troisième Italie est très différente de lâtalie du Nord-Ouest de lândustrie fordiste, et de lâtalie du Sud, où le processus dôndustrialisation est resté assez limité (Bagnasco, 1977). La troisième Italie est, en effet, la région post-fordiste par excellence. Son fonctionnement correspond au modèle de la spécialisation flexible (Piore, Sabel, 1984) : le processus productif peut être séparé en diverses phases, réparties entre des petites entreprises spécialisées. Il sâgit de productions sujettes à une variabilité élevée, aussi bien sur le plan quantitatif quầu niveau qualitatif.

La capacité exceptionnelle des districts industriels italiens à répondre de façon flexible aux transformations du marché se base certes sur lâısage de technologies nouvelles de la part des entreprises, mais surtout sur des relations horizontales entre les PMI : un certain équilibre se crée entre relations de coopération et de concurrence. La capacité à innover et à améliorer la qualité des biens est également soutenue par lâxxistence dốconomies externes (collaborateurs spécialisés, externalisations des services, infrastructures collectives) aux entreprises mais situées dans la même zone que les PMI. Cela permet la diffusion de lânformation et de lônnovation. A. Marshall parle à ce propos dô atmosphère indu strielle » (Marshall, 1900). Récemment, certains auteurs ont suggéré que lộn assistait dans le Mezzogiorno à lốmergence de formes de développement endogène similaires à celles de la troisième Italie, bien quấvidemment plus fragiles (Meldolesi, 1998 ; Rossi, 2004 ; Viesti, 2000).

Pour expliquer le dynamisme et le succès de ces systèmes productifs, les travaux mettent en exergue lômportance des facteurs locaux, et en particulier de la sédimentation dồne communauté locale en tant que ressource. Ce faisant, ils réintroduisent au cî ur de la réflexion la question du territoire et de la diversité

\footnotetext{
${ }^{1}$ Centre Robert Schuman pour les Etudes Avancées, Institut Universitaire Européen (Florence), camille.schmoll@iue.it.
} 
territoriale (Conti, Sforzi, 1997 ; Mariotti, 2004). De ce point de vue, ces travaux innovent par rapport à la littérature économique traditionnelle qui considérait que la spécificité du local - la « rugosité des lieux »- nôavait que peu de poids dans les choix de localisation et dans le développement économique.

La définition du territoire proposée dans ces travaux est très riche. Le territoire y est, tout dâbord, le lieu de sédimentation historique dôıne communauté et dồne culture : le développement des districts se base sur une «matrice » identitaire quâl sôngisse de la religion, de la politique ou de lâppartenance ethnique. Le territoire est également un lieu de proximité qui permet la relation, la contiguïté territoriale étant vue comme un moyen de relation et dấchange. Lâxistence dồn tissu dense de petites et moyennes villes, bien reliées entre elles, avec une distribution équilibrée sur le territoire de fonctions urbaines (banques, écoles, centres commerciaux, services) permet dôppuyer et de favoriser ces échanges (Bagnasco, 1999).

Cependant, lâpproche du territoire développée dans les travaux sur le développement local italien comporte certains écueils. En particulier, elle ne considère pas suffisamment le territoire comme une construction socio-spatiale processuelle, dynamique, soumise à dômportants changements dôrigine externe. La question de la mondialisation, par exemple, a certes souvent été abordée dans cette littérature, mais sous lôngle de lônternationalisation de la production et de la capacité dâxportation des districts, de leur compétitivité sur le plan international. Peu a été dit en revanche des transformations que connaissent les districts à lấchelle locale, ce qui conforte une idée assez statique des notions de territoire ou de développement local.

Dans les prochains paragraphes, jâessaierai de montrer comment les phénomènes de «mondialisation par le bas » (Portes, 1999), liés à la présence dântrepreneurs et dâemployés étrangers sur le territoire ont contribué à une reconfiguration des systèmes productifs locaux italiens. Je me concentrerai tout dâbord sur lấchelle nationale. Puis, dans un second temps, je montrerai, à partir de lâxemple du district vésuvien, situé en province de Naples, comment sêffectuent concrètement les interactions entre entreprises italiennes et populations étrangères.

Le district vésuvien demeure un exemple marginal au regard du dynamisme des districts de la troisième Italie : le travail au noir au sous-déclaré y est, par exemple, très présent ${ }^{2}$. De ce point de vue, il est clair que cet exemple ne pourra apporter que quelques éléments de réponse à la question du lien entre développement local et migrations internationales. Cependant, il présente un intérêt certain puisque, comme il a été dit plus haut, certains travaux ont reconnu dans les systèmes productifs locaux du Mezzogiorno des éléments vertueux rappelant le modèle du district. De plus, le district vésuvien, à la différence des districts de lâtalie centrale et septentrionale, sâst développé assez récemment, en même temps quâıne présence étrangère sôaffirmait dans la zone: il constitue donc un exemple particulièrement intéressant de développement local puisquôl a attiré et a du intégrer dans son fonctionnement économique des migrants étrangers dès les premières phases de son développement, au cours des années 80 .

\footnotetext{
${ }^{2}$ La définition même de district pourrait être contestée bien que lâxistence du district vésuvien ait été reconnue par la loi régionale $n .17$ de 1991 (sur la question de la définition des districts, on peut lire la contribution dôAlessia Mariotti et Paolo Molinari dans ce numéro). Pour une approche critique à la question du développement local dans le Mezzogiorno voir Rossi, 2004 ; Dunford, Greco, 2006.
} 


\section{Districts industriels et main dô̂ uvre étrangère en Italie}

La littérature sur les districts industriels ne fait généralement référence aux flux migratoires que pour représenter les migrants comme une menace à lấquilibre économique local. Cette menace est souvent incarnée dans la figure de lậntrepreneur chinois : lâxxemple de Prato, symbole du développement local à lâtalienne, et en même temps un des principaux pôles dônstallation des Chinois en Italie ${ }^{3}$, est souvent évoqué pour montrer que la venue des Chinois a contribué au déclin du district, en irriguant le marché local de produits de basse qualité. Cependant, pour Prato comme pour dôutres villes dâtalie, la présence dône main dố uvre chinoise à bas coût a pu représenter, du moins dans les premiers temps de leur installation, des complémentarités avec lấconomie locale. La question de la concurrence que représentent les entreprises chinoises est donc probablement plus complexe quâlle nôpparaît au premier abord. Les entrepreneurs des systèmes productifs locaux, en effet, ne sont pas seulement victimes de phénomènes de «mondialisation par le bas », ils en sont aussi des acteurs, en créant une demande dêmploi en sous-traitance.

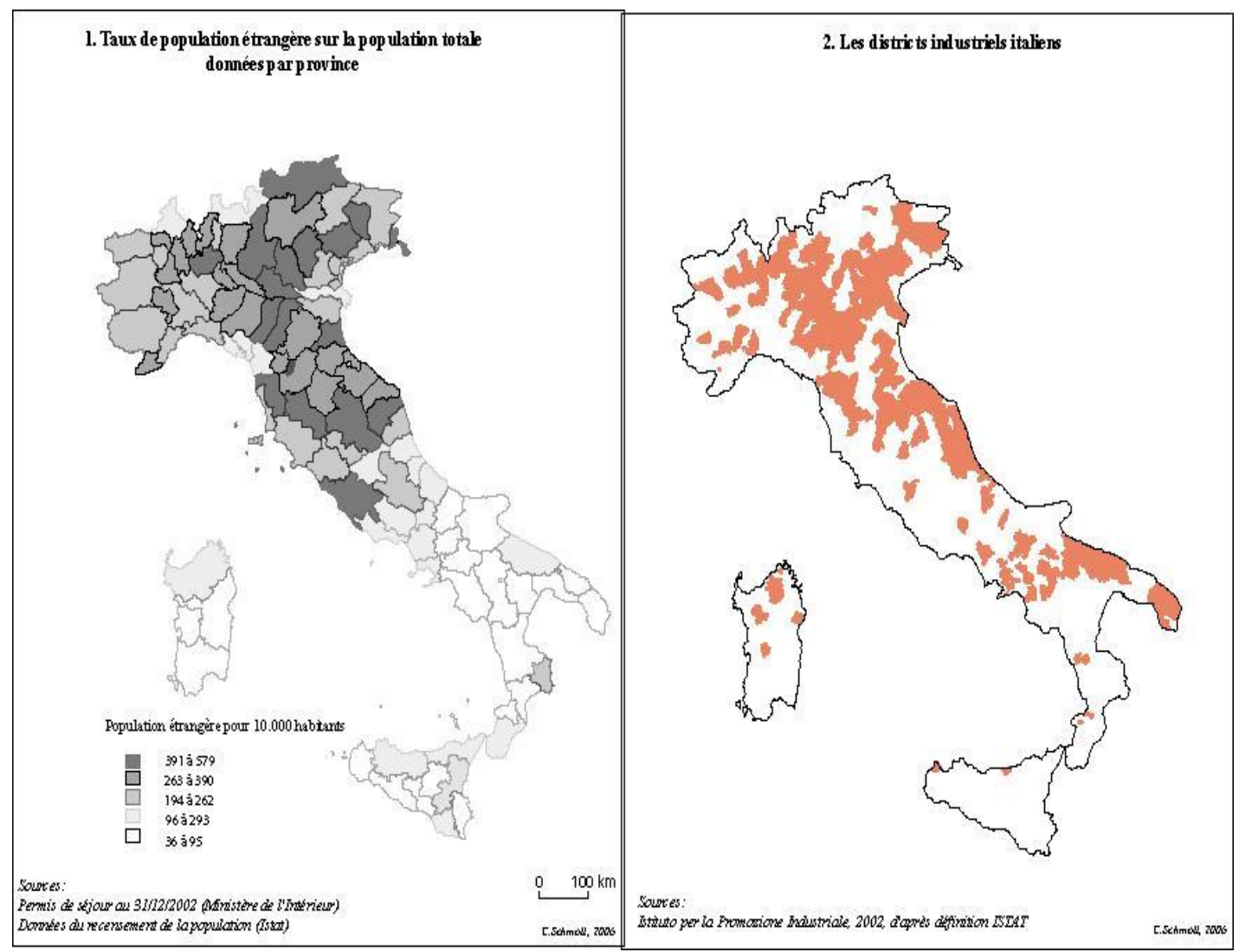

\footnotetext{
${ }^{3}$ Les Chinois, à Prato, représentent presque 4\% de la population résidente, soient 6813 habitants sur un total de 108600 habitants (Istat, 2004).
} 
Les étrangers représentent aujourdôhui plus de 4,5\% de la population de lâtalie. Leur répartition sur le territoire italien montre que les principaux espaces dônstallation sont, outre les grandes agglomérations, les districts industriels. La corrélation entre la localisation des districts industriels et celle des populations étrangères en Italie, quôn observe sur les cartes 1 et 2, peut facilement être expliquée : les étrangers représentent, pour les entrepreneurs des systèmes productifs locaux italiens, une ressource de toute première importance. Il sâgit, en premier lieu, dôouvriers qui constituent une main dỗ uvre flexible et bon marché, au regard de la main dỗ uvre locale. A ce sujet, certains auteurs signalent la mise en place de canaux de recrutement communautaires, basés sur un accord informel entre lâentrepreneur et son ouvrier, ce dernier assurant un rôle dôntermédiaire entre le chef dêntreprise et ses compatriotes (Ambrosini, 2001 ; Palidda, 2002). Il convient également de souligner lâmportance croissante, dans ces zones productives, de femmes migrantes, provenant souvent dôEurope centrale et orientale, employées dans des activités de travail domestique (travaux ménagers, soins aux enfants et aux personnes âgées). On assiste ainsi, dans les districts industriels, à la mise en place dôn véritable marché du travail segmenté selon lâppartenance ethnique et sexuée des individus. Les femmes migrantes sont chargées des travaux traditionnellement dévolus aux femmes italiennes qui, du fait de leur entrée sur le marché du travail et dôn processus plus général dấmancipation, se refusent désormais dêffectuer ces tâches, du moins dans leur totalité (Mingozzi, 2005, Miranda, 2003). Câest donc tout le «système travail/famille», dans les districts industriels, qui repose sur la main dỗ uvre étrangère (Pleck, 1977).

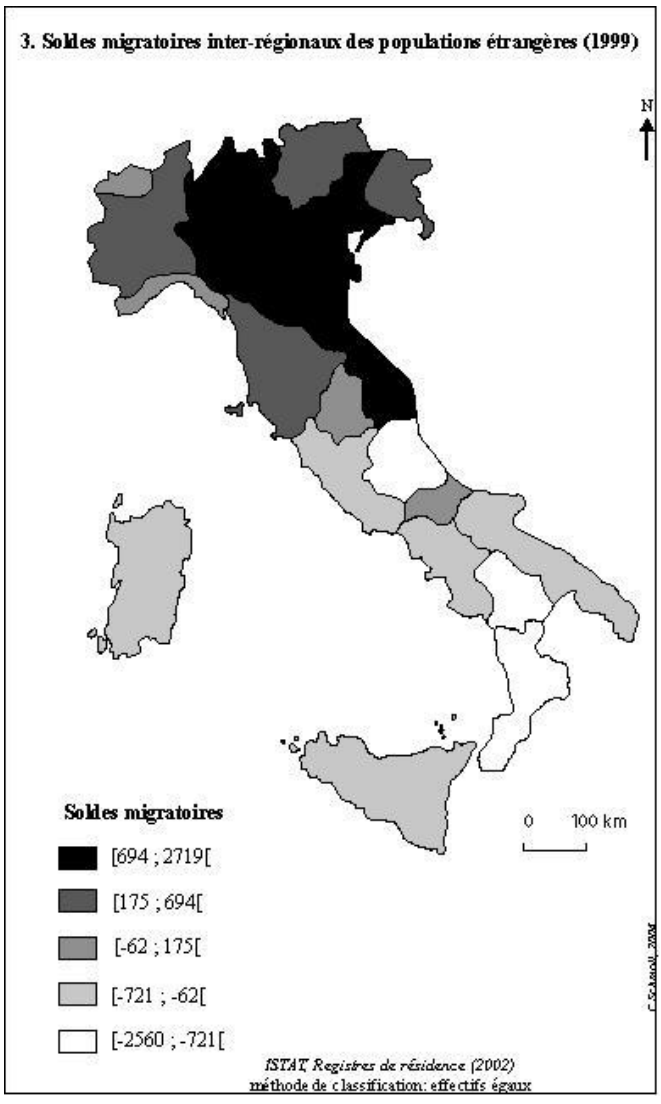


Lônstallation des populations étrangères dans ces régions productives est souvent le résultat dôın processus de mobilité spatiale du Sud vers le Nord de lâtalie, à la suite dôıne opération de régularisation. La trajectoire-type est la suivante : les migrants, irréguliers ou clandestins, passent les premiers temps de leur carrière migratoire dans les régions du Centre-Sud, où la tolérance à lấgard de lấtranger est majeure, du fait dôıne économie souterraine diffuse et largement tolérée. Dans un second temps, ils se déplacent vers le Nord. La carte n.3, qui représente les soldes migratoires interrégionaux sur une année suivant une période de régularisation (1999) illustre bien ce phénomène de mobilité seconde, notamment en direction des régions du Nord-est.

\section{Le développement du district vésuvien}

Ainsi, lấconomie des districts se base sur une importante main-dôeuvre étrangère. Le groupement de communes correspondant au district vésuvien, dont lấpicentre est la commune de San Giuseppe Vesuviano, est exemplaire à cet égard ${ }^{4}$. Le district vésuvien présente des taux de population étrangère particulièrement élevés par rapport au reste ede la province d Naples, comme on peut le voir sur la carte n. 4.

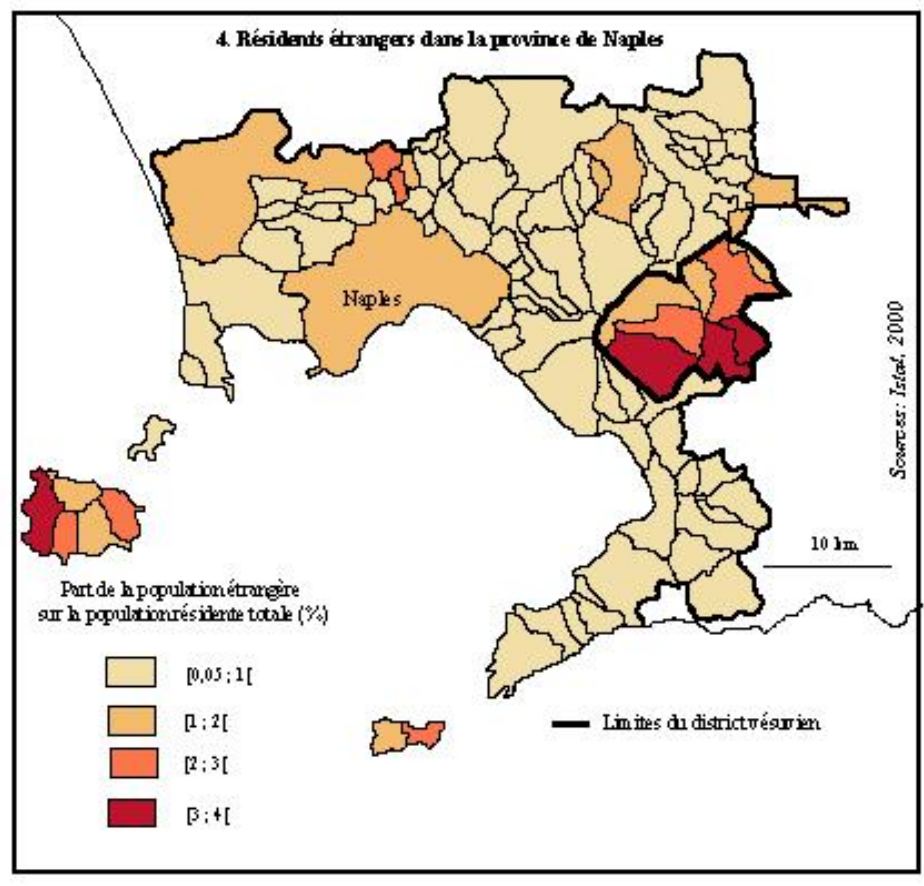

\footnotetext{
${ }^{4}$ Le district vésuvien comprend les communes de Carbonara di Nola, Ottaviano, San Gennaro Vesuviano, San Giuseppe Vesuviano, Palma Campania, Poggiomarino, Striano et Terzigno. Lâxemple du district vésuvien a été présenté plus en détail dans Schmoll, 2001.
} 
La vocation textile de San Giuseppe Vesuviano prend naissance dans lâprès-guerre, quand les habitants de San Giuseppe se spécialisent dans la vente au porte-à-porte de produits textiles. Dans les années 70, certains dâentre eux investissent les fonds accumulés dans des points de vente en gros, qui leur permettent de jouer un rôle dôntermédiaires entre les districts productifs du Nord et les commerces de détail du Mezzogiorno. Puis, au cours des années 80, les grossistes de San Giuseppe décident de se lancer dans la production textile afin de sôffranchir de leur dépendance structurelle vis-à-vis des régions septentrionales (Aniello, Meldolesi, 1998). Un secteur productif, spécialisé dans lôhabillement et le linge de maison, organisé en fabriques sous-traitantes de petite taille (les fabrichette) généralement non déclarées, se développe alors dans toute la zone vésuvienne. Au départ, la production se base essentiellement sur une main dỗ uvre familiale. Cependant, dès la fin des années 80, alors que les flux migratoires à destination de lâtalie se renforcent, une main dỗ uvre masculine en provenance du Maghreb, attirée par le développement du district, commence à travailler dans les fabrichette.

Ces nouveaux arrivants ne sont pas tous ouvriers : certains Marocains reprennent la tradition de colportage désormais abandonnée par les entrepreneurs locaux enrichis, et deviennent ainsi clients des producteurs-grossistes de San Giuseppe. Armés dồn carrozzino, poussette pour enfants aménagée en porte-marchandises, ils arpentent lâgglomération napolitaine et proposent, au porte-à-porte, les marchandises produites dans le district vésuvien.

Progressivement, le district devient un centre régional dâpprovisionnement pour des petits commerçants de rue étrangers affluant de tout le Mezzogiorno. Surtout, le marché vésuvien devient un pôle dôchat pour des populations circulantes maghrébines, de provenances et de statuts diversifiés, qui sâpprovisionnent en vêtements et en linge de maison pour les revendre dans dôutres villes dôEurope ou dans leurs lieux dôrrigine au Maghreb. Il sôntègre ainsi dans des économies circulatoires maghrébines qui traversent lấspace euro-méditerranéen et comprennent un ensemble de lieux : quartiers urbains, zones productives, centrales de vente en gros et ports (Péraldi, 2001 ; Tarrius, 1995). Il sôgit également dônn lieu dôachat pour des migrants de retour au pays, qui souhaitent rapporter des produits made in Italy à leur entourage. Les produits de linge de maison et de lingerie du district vésuvien, qui constituent de parfaits cadeaux de mariage, sont particulièrement appréciés.

Cette clientèle maghrébine est loin dộtre négligeable pour les grossistes vésuviens, comme en témoignent de nombreux indices (présence de commis arabophones dans les boutiques, diffusion de stratégies de marketing et de visibilité en arabe telles que la traduction des enseignes, la publication dônnonces dans des journaux spécialisés). Au cours des années 90, San Giuseppe connaît un développement remarquable et devient une des communes les plus riches de Campanie (Biondi, 2000). Il nâst, dans ce contexte, guère surprenant que le phénomène de la «bonne » polonaise ou ukrainienne sôy soit développé assez rapidement : chaque famille de San Giuseppe compte désormais au moins une domestique originaire dôEurope centrale ou orientale. Surtout, câst à cette époque que lântrepreneur vésuvien, désireux de comprimer les coûts de production, fait appel à lântreprise sous-traitante chinoise, si bien que lôarea vesuviana constitue aujourdôui le premier point dôncrage des Chinois en Campanie. Les entrepreneurs chinois mettent en place des structures productives, qui utilisent une main dỗ uvre communautaire et font converger successivement vers San Giuseppe une partie des flux migratoires des années 90. Ainsi, les Chinois conquièrent petit à petit le secteur de la production textile dans lôarea vesuviana. 
Au départ, lântreprise chinoise, au sein du système de San Giuseppe, ne contrôle ni lômont, ni lâval du cycle et dépend entièrement des commandes des entrepreneursgrossistes italiens. Cependant, lôouverture, depuis 1998, dône centaine de grossistes chinois en habillement (vêtements importés de Chine) dans lâirea vesuviana a renforcé lôutonomie des Chinois vis-à-vis des grossistes italiens, qui sônquiètent de cette concurrence. Le développement de la vente de produits chinois a, quoi quôl en soit, contribué à renforcer le pouvoir attractif du pôle vésuvien auprès des populations maghrébines qui, sans pour autant délaisser les produits made in Italy, semblent apprécier la diversité de lôffre commerciale vésuvienne.

\section{Conclusion}

Lâxemple du système vésuvien témoigne de lâptitude de lântreprise « ethnique » à influer sur le développement local, à la fois en amont et en aval. Toutefois, on manque cruellement de travaux permettant dêxpliquer plus précisément les relations économiques qui sấtablissent entre entreprises italiennes et populations étrangères sur le territoire italien. Les exemples évoqués dans ce texte montrent, quoi quâl en soit, que le migrant étranger est un acteur central du tissu productif italien : en tant quôuvrier et quântrepreneur, en tant que soutien à lấconomie domestique, en tant que clientèle. Il ne peut donc plus être considéré comme un élément externe et un simple facteur de concurrence, puisquôon observe de multiples formes de complémentarité économique entre étrangers et Italiens. Cela montre également que la relation entre migrations internationales et tissu économique est devenue autrement plus complexe quầ lấpoque fordiste. De ce point de vue, lâtalie, devenue récemment un pays dômmigration massive, est un exemple tout a fait passionnant pour étudier la relation entre post-fordisme et migrations internationales (Schmoll, Weber, 2004).

\section{Bibliographie}

Ambrosini M., 2001, La fatica di integrarsi, Bologne, Il Mulino.

Aniello V., Meldolesi L. (dir.), 1998, double numéro spécial ñLâtalia che non cấ. Quantồ. Dovâ.. Comầ.ò, Rivista di Politica Economica, 8-9, 10-11, août-septembre, octobre-novembre.

Bagnasco A., 1977, Tre Italie, Bologne, Il Mulino.

Bagnasco A., 1999, Tracce di comunità, Bologne, Il Mulino.

Biondi G. (dir.), 2000, Industria e territorio, Camera di Commercio Industria Artigianato e Agricoltura di Napoli, Naples, Unione degli Industriali della Provincia di Napoli.

Conti S., Sforzi F., 1997, ñll sistema produttivo italianoò, Coppola P. (dir.), Geografia politica delle regioni italiane, Turin, Einaudi, pp. 278-336.

Dunford M., Greco L., 2006, After the three Italies. Wealth, inequalities and industrial Change, Oxford, Blackwell.

Mariotti A., 2004, «Lôndustrie italienne : dôıne lecture économique à lune lecture territoriale », Vallat C. (dir.), Autres vues dôtalie. Lectures géographiques dôın territoire, Paris, lâHarmattan, pp. 259-275.

Marshall A., 1900, Elements of Economics of Industry, Londres, Macmillan.

Meldolesi L., 1998, Dalla Parte del Sud, Rome-Bari, Laterza.

Mingozzi A., 2005, ก̃ll lavoro domestico nel distretto faentino. Effettti della regolarizzazione sulle lavoratrici proveniente dallâx Unione Sovieticaò, Colombo A., 
Caponio T. (dir.), 2005, Stranieri in Italia. Migrazioni globali, integrazioni locali, Bologne, Il Mulino, pp. 117-143.

Miranda A., 2003, ñDomestiche straniere e datrici di lavoro autoctone. Un incontro culturale asimmetricoò, Studi Emigrazione, 148, pp. 859-879.

Palidda S., 2002, ñPasseurs, Mediatori, Intermediariò, La Ricerca Floklorica, 44, pp.77-84.

Péraldi M. (dir.), 2001, Cabas et containers. Activités marchandes informelles et réseaux migrants transfrontaliers, Paris, Maisonneuve et Larose.

Piore M., Sabel C., 1984, The second industrial divide, New York, Basic Books.

Pleck J.H., 1977, ñThe work-family role systemò, Social Problems, 24, pp. 417-427.

Portes A., 1999, "La mondialisation par le bas. Lốmergence des communautés transnationales », Actes de la recherche en sciences sociales, 129, pp. 15-25.

Rossi U., 2004, ñNew Regionalism contested. Some remarks on the case of the Mezzogiorno of Italyò, International Journal of Urban and Regional Research, 28, 2, pp.466-476.

Schmoll C., 2001, ñImmigration et nouvelles marges productives dans lâaire métropolitaine de Naplesò, Bulletin de lôAssociation des géographes de France, 4, pp. 403-413.

Schmoll C., Weber S., 2004, «Un laboratoire dômmigration post-fordiste », Vallat C. (dir.), Autres vues dôtalie. Lectures géographiques dồn territoire, Paris, lôHarmattan, pp. 125-167.

Tarrius A., avec la collaboration de L. Missaoui, 1995, Arabes de France dams lấconomie mondiale souterraine, Paris, Editions de lôAube.

Viesti G., 2000, Mezzogiorno dei distretti, Rome, Donzelli. 